\title{
Une invitation au tourisme : l'affiche ferroviaire française (1880-1936)
}

Nathalie Pégé-Defendi

\section{CpenEdition}

\section{Journals}

Édition électronique

URL : https://journals.openedition.org/rhcf/1914

DOI : 10.4000/rhcf.1914

\section{Éditeur}

Rails \& histoire

\section{Édition imprimée}

Date de publication : 1 juin 2003

Pagination : 27-38

ISBN : 0996-9403

ISSN : 0996-9403

\section{Référence électronique}

Nathalie Pégé-Defendi, « Une invitation au tourisme : l'affiche ferroviaire française (1880-1936) », Revue d'histoire des chemins de fer [En ligne], 27 | 2003, mis en ligne le 15 janvier 2015, consulté le 22 avril 2022. URL : http://journals.openedition.org/rhcf/1914 ; DOI : https://doi.org/10.4000/rhcf.1914

Ce document a été généré automatiquement le 22 avril 2022

Tous droits réservés 


\title{
Une invitation au tourisme : l'affiche ferroviaire française (1880-1936)
}

\author{
Nathalie Pégé-Defendi
}

1 Partant du postulat que l'image participe à part entière de la connaissance des faits historiques, nous nous sommes appliqués, dans le cadre d'une contribution à l'histoire du marketing touristique, à décrire la création et la réception de l'affiche ferroviaire française de 1880 à 1936. A travers ce cas particulier, il s'agissait d'approfondir l'histoire des facteurs de production de la promotion touristique des stations, des comportements qu'ils conditionnent, des coûts qu'ils déterminent ${ }^{1}$.

2 Choisir une image fixe comme sujet de recherche n'est pas neutre et induit une somme de questions quant au statut de celle-ci, au type de sources que constituent ces documents figuratifs : il s'agit du crédit à leur accorder pour la connaissance du passé. Les images ont été considérées trop longtemps par les historiens comme simples illustrations, c'est-à-dire comme un agrément qui venait corroborer l'écrit, auquel ils déniaient la qualité de source à part entière. Or, les images peuvent devenir de véritables sujets d'études en elles-mêmes. La prise en compte de corpus (ensembles) d'images, le souci de la cohérence et du choix de ces corpus motivent désormais certains disciples de Clio. On peut ainsi qualifier de réconciliation entre histoire et histoire de l'art la vague récente de ces études sur l'image. Si le scepticisme moderne a, de même, encouragé les historiens à considérer les arts avec plus de bienveillance, les temps y ont été propices. La facilité des voyages, le nombre sans cesse croissant de musées et d'expositions, l'ouverture de demeures privées et de collections et, par-dessus tout, l'amélioration des techniques de reproduction photographique ont facilité plus que jamais auparavant une connaissance directe des arts plastiques par les historiens.

3 La collection d'affiches présente certaines difficultés, dues à leurs caractères propres. Tirée à de nombreux exemplaires au moment où elle est placardée sur les murs, l'affiche est doublement éphémère, par la nature transitoire de l'événement qu'elle est chargée d'évoquer comme par la brièveté de son exposition, de sa durée. Son rôle est 
d'informer mais aussi de promouvoir et pour cela elle utilise divers moyens de persuasion dont celui de l'art, de la jouissance esthétique. Informative, l'affiche adopte volontiers un langage caractérisé par la déformation grossissante, le slogan idéologique. Mais le langage de l'affiche peut aussi se rapprocher de celui du photographe et de son pouvoir de suggestion. Art de la rue, l'affiche typographique ou illustrée a suivi les modes et les courants artistiques.

4 Notre projet devait, pour fonder une typologie de l'affiche ferroviaire française, s'asseoir sur un corpus important d'affiches. Nous avons bâti pour la période 1880-1936 un corpus de 1684 affiches (illustrations différentes), ce qui représente l'essentiel des collections conservées. Dès 1880 , le phénomène touristique est bien installé, une publicité se révèle donc indispensable, les moyens relativement modestes utilisés jusque-là atteignent leur limite. Ces années correspondent aux débuts de la propagande ferroviaire touristique et à la mise en place, pour la première fois en France, d'une véritable politique touristique concertée et à la professionnalisation du monde du tourisme.

5 La naissance de l'affiche est donc due à la concurrence effrénée que se livrent les stations touristiques et les réseaux ferroviaires qui les desservent; elle est rendue possible par la mise au point de la lithographie. Cette affiche ferroviaire française est d'abord et avant tout une affiche touristique. Elle vante les destinations touristiques. Mais, pour être ferroviaire et entrer dans notre corpus, elle doit correspondre à certains critères. En premier lieu, doit figurer le nom d'une compagnie de chemin de fer, sous forme de bandeau, écrit dans le texte, sous forme de logo, de macaron... Dans les années 1920 , son format se standardisa $(60 \times 120 \mathrm{~cm})$.

6 Ainsi l'étude de la conception de l'affiche ferroviaire nous a permis de pénétrer le monde ferroviaire et de connaître l'organisation des réseaux en matière de développement de leur trafic touristique. La question était de savoir si la notion de tourisme avait évolué dans le temps.

7 Les réseaux ont très tôt réfléchi à une politique commerciale pour leurs lignes touristiques. Leur première action fut de multiplier les tarifs spéciaux et attractifs dès les années 1850. Elle fut suivie ensuite d'actions touristiques multiformes: trains spéciaux pour les lignes touristiques, offre combinée de services de transport automobile, d'hôtels... L'ultime étape fut la nomination d'un responsable de la publicité dans chaque réseau, chargé de mettre sur pied une véritable politique commerciale.

8 L'étude de l'émergence de cet homme de publicité nous a permis de nous pencher sur les rapports entretenus entre les réseaux et la publicité, nous offrant la possibilité d'une relecture du marketing publicitaire des réseaux et la possibilité de s'interroger à nouveau sur la thèse d'un « retard français » en matière de publicité et de marketing.

9 Le monde ferroviaire privilégie le technique, la voie, le matériel et les flux au détriment de l'exploitation. En effet, sous l'influence des ingénieurs, les réseaux privilégient les problèmes de lignes ou d'organisation, au détriment de ceux de la vente. Il n'est donc pas surprenant que la publicité leur paraisse un élément secondaire. La méfiance à l'égard de la publicité ne favorisait pas non plus la création de services qui lui soient dédiés; néanmoins, les réseaux y vinrent progressivement.

Ainsi nous avons pu étudier l'ensemble des supports publicitaires, établir leurs coûts et, notamment, la place qu'y tient l'affiche. Des premières petites annonces informatives sur papier de couleur indiquant la date et l'heure des premiers trains, les réseaux sont 
très vite passés à une production très variée de supports publicitaires multiformes : affiches, guides, agendas, films, émissions radiophoniques... L'affiche était très appréciée comme un media souple qui permet facilement de fractionner dans le temps les dépenses qu'il occasionne et qui laisse les annonceurs maîtres de leur campagne. Plusieurs éléments laissent à penser que l'affiche ferroviaire n'a pas été créée à une période donnée, mais que les affiches de renseignements ferroviaires se sont peu à peu illustrées, pour finalement n'être plus que des affiches ferroviaires illustrées. La décision de faire réaliser une affiche pouvait être suivie d'une commande, qu'il s'agisse d'un concours ou non, d'une collaboration avec le milieu du tourisme ou d'une collaboration, ou non, avec les autres réseaux. La question d'argent fait souvent préférer dans un premier temps la presse, puis l'affiche à tout autre support. Plus tard, on l'a vu, dans les années 1920-1930, les nouveaux supports modernes comme la TSF supplantèrent la vieille affiche au goût devenu suranné. En effet, dans cette période d'avant-guerre, nous avons assisté à une transformation des mentalités vis-à-vis de la publicité. Elle paraît obligatoire pour les uns et une chance pour les autres. Les actions se radicalisent et se font plus systématiques.

11 Initialement, les budgets publicitaires n'existaient pas. Ils furent systématiques dans les années 1920, pour prendre une part importante dans les années 1930, avec de grandes disparités entre les réseaux. A ces budgets directement affectés à la publicité, il faut ajouter les montants des subventions allouées par les compagnies au monde du tourisme: au niveau local par des subventions aux syndicats d'initiative, au niveau régional par des subventions aux fédérations des syndicats d'initiative et à l'échelon national par des subventions à l'office national du tourisme. On retrouve ici le rôle déjà ancien des compagnies qui accordaient très souvent des subventions à différentes associations, pour diverses manifestations en rapport ou non avec la compagnie: courses, foires, symphonies... Ces nombreuses manifestations subventionnées étaient l'occasion pour les compagnies de propagande gratuite de leurs lignes. Toutes les actions engagées par les réseaux s'inscrivaient dans une véritable stratégie de propagande commerciale par le tourisme et pour le tourisme.

12 Par ailleurs, les compagnies ayant compris très vite la rentrée d'argent que pouvait représenter la vente d'affiches et de cartes postales, elles ont cherché à les vendre au grand public, par voie de presse ou en s'associant avec des prestataires.

13 Cette association entre les réseaux et le monde du tourisme était très efficace dans la création des affiches, même si les relations qu'entretenait le milieu ferroviaire avec le monde du tourisme et celui de l'impression oscillaient entre rapports de force, mépris et collaboration. En règle générale, l'office du tourisme ou le service municipal dédié à cette activité avait un projet d'affiche, ou avait déjà fait réaliser l'affiche et demandait de l'argent à la compagnie qui desservait la station. La compagnie n'accordait ou non cette subvention qu'après mûre réflexion et étude de la maquette. Les réseaux n'apposaient gratuitement dans leurs gares et dans leurs voitures des affiches ou des pancartes qu'après avoir bien considéré l'affiche en question. Comme nous l'avons constaté, ils choisissent souvent les artistes qui les réalisent ou, tout au moins, ils donnent des consignes précises pour leur réalisation. Il faut en effet que l'affiche revête un intérêt touristique réel et seconde ainsi le mieux possible l'action de propagande des réseaux. Même si les retombées économiques induites par ces affiches n'ont pas été quantifiées, ni par les réseaux, ni par les syndicats d'initiative, elles sont indéniables. 
14 L'idée de faire réaliser des affiches émanait donc tout d'abord des milieux touristiques, relayés ensuite par les réseaux. L'affiche ferroviaire était pour les offices du tourisme le support publicitaire prioritaire sur tout autre support, avec la presse. La décision de faire exécuter une affiche pour la promotion de la station peut être le résultat de longues réflexions, comme le fruit du hasard : l'air du temps, l'habitude, la proposition d'un imprimeur, la réponse à une affiche de la station voisine et néanmoins concurrente et, quelquefois, tout cela à la fois. Les concours permettaient aussi aux syndicats d'initiative de se procurer des affiches à un coût réduit, en achetant des œuvres non primées. Mais l'abus des concours finit par conduire les meilleurs artistes à ne plus y participer car les résultats en étaient fort aléatoires.

Ainsi, une action importante s'exerça à l'intérieur de la France pour diffuser des documents de publicité. Deux milieux, aux intérêts convergents, se sont réunis pour mener à bien cette tâche : le milieu ferroviaire et le monde du tourisme.

A partir des années 1880 , le monde de l'imprimerie se développe. Un des facteurs déterminants dans ce développement des imprimeries est l'assouplissement de la législation qui encadre leur création. La loi de 1881 abroge toute la législation antérieure spécifique à l'imprimerie et à la presse (quarante-deux lois, décrets et ordonnances). En 1881, l'imprimeur (250 ont été repérés dans le corpus) est un entrepreneur, fournisseur de forces productives (main-d'œuvre, outillage, machines) qui permettent de transformer un concept personnel en facture, affiche, prospectus, brochure, livre...

17 La mise au point de la lithographie est un progrès décisif qui bouleverse les habitudes et modernise la réclame touristique. Cette nouvelle technique modifie les rapports professionnels, l'illustration devenant prépondérante sur le texte. Le dessinateur, l'artiste (500 dans le corpus), portent la responsabilité générale de l'affiche. Ils la conçoivent selon les directives du commanditaire, et laissent si nécessaire une place pour le texte que le typographe remplit tant bien que mal.

18 L'analyse du corpus a montré que la grande majorité des affiches ( $80 \%)$ était de style figuratif que nous dirons traditionnel contre $20 \%$ de style graphique et ce, même audelà des années 1910-1920. Pourquoi la France était-elle attachée à cette conception traditionnelle de l'affiche? La première raison avancée est la réaction de rejet quasi unanime de la classe dirigeante à l'égard de la publicité que nous avons déjà citée, son conservatisme et ses réticences devant les audaces modernes, d'influence allemande. N'oublions pas le rôle pédagogico-paternaliste qu'entendaient jouer les réseaux via leurs affiches, en faisant des salles d'attente des gares une sorte de petit musée de peinture voué à l'instruction du public et par l'envoi massif d'affiches dans les lieux publics. Autre raison à cette tendance, la formation académique des illustrateurs et les influences artistiques subies par ceux-ci.

Le PLM est la compagnie qui a fait réaliser le plus d'affiches : près de $30 \%$ du corpus, suivi par le Nord (21\%) et le PO (11,97\%) (Annexe 1, tabl. 1).

20 Le premier élément d'accroche publicitaire utilisé par le PLM et l'État était le paysage, alors que le Nord et l'Ouest préféraient mettre en avant des bourgeoises souriantes ou des femmes en habit folklorique. Les autres compagnies n'ont pas utilisé de façon notable un élément de préférence à un autre. Le style propre à chaque compagnie est à rechercher dans le choix de l'imprimeur et de l'artiste (Annexes 2 et 3, tabl. 2 et 3). 
21 L'interrogation sur la place de l'affiche dans l'environnement urbain offre aussi la possibilité de considérer les images non seulement comme le travail d'artistes, mais aussi comme des éléments perturbateurs et actifs. Les affiches n'ont, en effet, pas le même impact sur les badauds, les défenseurs de la nature, les publicitaires, les critiques d'art... Ainsi, les réglementations de l'affichage peuvent être lues comme des réponses à ces perturbations et, donc, font partie des paramètres à prendre en compte dans l'étude, au même titre que le format de l'image placardée, son support, sa distribution dans l'espace urbain, la fiscalité qui la concerne, sa place dans la littérature et dans la presse.

Le développement du tourisme n'a pu se faire sans la promotion d'images régionales fortes. Les premières images attirantes des régions offrirent une représentation exotique et pittoresque que diffusèrent les peintres, les écrivains et les érudits locaux. Elle se mua par la suite en un stéréotype que reprirent chansonniers, romanciers, publicitaires et acteurs économiques du tourisme. Tous semblaient présenter «ces pays ", figés dans un folklore parfois inventé pour l'occasion. Le costume et la coiffe suffirent rapidement à symboliser pour un large public une image familière. Le spectacle des fêtes locales, les produits de l'industrie du souvenir contribuèrent à renforcer la vision passéiste et nostalgique des régions alors que les guides touristiques transformèrent la dure réalité quotidienne des populations en scènes pittoresques.

L'affiche ferroviaire participa ainsi à l'évolution de la perception des éléments naturels et régionaux et, par là même, à la construction des mythes fondateurs du tourisme.

Illustrateur : Landrieux - Commanditaire : Chemin de fer du Nord

\section{ANNEXES}

Annexe 1 
Tableau 1. La production d'affiches ferroviaires, 1830-1936. Nombre d'affiches par compagnie.

\begin{tabular}{|l|c|r|}
\hline Cies ferroviaires & Nombre d'affiches & $\%$ \\
\hline PLM & 546 & 28,54 \\
\hline Nord & 202 & 21,01 \\
\hline Ouest & 100 & 5,23 \\
\hline État & 222 & 11,60 \\
\hline Est & 87 & 4,55 \\
\hline PO & 229 & 11,97 \\
\hline PO-Midi & 69 & 3,61 \\
\hline Midi & 117 & 6,12 \\
\hline AL & 48 & 2,51 \\
\hline Gds Réseaux français & 25 & 1,31 \\
\hline Autres & 67 & 3,50 \\
\hline SNCF & 1 & 0,05 \\
\hline
\end{tabular}

Compagnies ferroviaires

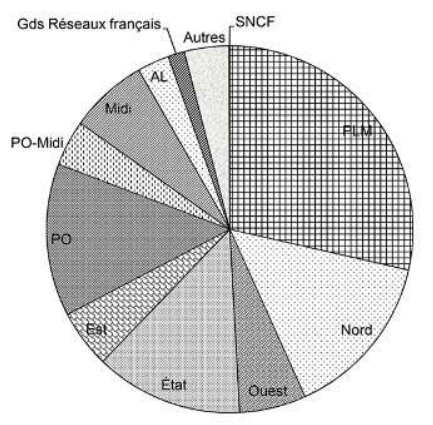

Certaines affiches sont citées plusieurs fois car des compagnies se sont associées pour les créer, ce qui explique que le nombre total des affiches produites dépasse le nombre total des affiches du corpus étudié.

\section{Annexe 2}


Tableau 2. Association entre illustrateurs et compagnies ferroviaires, 1880-1936.

\begin{tabular}{|c|c|c|c|c|}
\hline NOM & $\begin{array}{c}\text { Nbre } \\
\text { d'affiches }\end{array}$ & $\begin{array}{c}\text { Nbre de } \\
\text { Cies }\end{array}$ & $\begin{array}{l}\text { Cie principale } \\
\text { d'affiches }\end{array}$ & \\
\hline ALESI Hugo d'-F. & 82 & 6 & PLM & 136 \\
\hline ALO & 84 & 9 & Orléans & 130 \\
\hline BOURGEOIS Eugène & 14 & 5 & Ouest/Est/PLM & 14 \\
\hline BRODERS Roger & 73 & 5 & PLM & 164 \\
\hline CASSANDRE & 13 & 5 & Nord & 18 \\
\hline CHAMPSEIX E.-Paul & 23 & 6 & Midi & $/ 16$ \\
\hline CHERET Jules & 13 & 5 & PLM & 17 \\
\hline COMMARMOND Pierre & 39 & 9 & Midi & 18 \\
\hline CONSTANT DUVAL & 46 & 6 & Orléans & 125 \\
\hline DORIVAL Géo & 44 & 12 & PLM & $/ 14$ \\
\hline FIX-MASSEAU Pierre & 10 & 5 & État & 14 \\
\hline FRAIPONT Gustave & 51 & 8 & Ouest & $/ 17$ \\
\hline GRAY Henri & 13 & 5 & Nord & 19 \\
\hline HUGON Roland & 10 & 4 & SNCF & 15 \\
\hline LACAZE Julien & 45 & 8 & PLM & 124 \\
\hline NEZIERE J. de la & 10 & 2 & PLM & 19 \\
\hline PEAN René-Louis & 16 & 5 & Ouest & 16 \\
\hline SOUBIE Roger & 19 & 6 & PLM & 110 \\
\hline TANCONVILLE Henri & 26 & 2 & PLM & 123 \\
\hline TAUZIN Louis & 18 & 6 & PLM & 15 \\
\hline TOUSSAINT Maurice & 14 & 5 & État & 15 \\
\hline $\begin{array}{l}\text { TRINQUIER-TRIANON } \\
\text { L. }\end{array}$ & 16 & 3 & PLM & 110 \\
\hline
\end{tabular}

L'échantillon retenu est composé des 22 illustrateurs ayant réalisé plus de 10 affiches chacun pendant la période étudiée. On constate que, sur les 679 affiches du corpus correspondant, 206 étaient libellées PLM, soit plus de $30 \%$. Si l'on excepte quelques illustrateurs qui ont travaillé pour 3 compagnies au plus, on peut dire que les illustrateurs, en général, ont privilégié un commanditaire sans en exclure aucun. On ne peut donc pas parler d'exclusivité entre un auteur et un réseau, sauf pour une période donnée, si elle est par exemple fixée par contrat.

\section{Annexe 3}


Tableau 3. Association entre imprimeries et compagnies ferroviaires, 1880-1936.

\begin{tabular}{|c|c|c|c|c|c|}
\hline & NOM & Nbre d'affiches & Nbre de Cies & \multicolumn{2}{|c|}{ Cie principale et nbre d'affiches } \\
\hline 2 & Imprimerie Serre & 325 & 15 & PLM & $/ 121$ \\
\hline 3 & Imprimerie Chaix & 89 & 16 & Ouest & 124 \\
\hline 4 & Imprimerie F. Champenois & 87 & 12 & Orléans & $/ 27$ \\
\hline 5 & Imprimerie Courmont Frères & 56 & 11 & PLM & $/ 18$ \\
\hline 6 & Imprimerie Hugo d'Alési & 54 & 5 & PLM & 142 \\
\hline 7 & Imprimerie F. \& M. Moreau & 36 & 7 & Orléans & $/ 16$ \\
\hline 8 & Imprimerie L. Danel & 26 & 9 & Nord & 120 \\
\hline 9 & Imprimerie Moullot fils aîné & 22 & 5 & PLM & 120 \\
\hline 10 & Imprimerie G. de Malherbe \& Cellot & 19 & 4 & Orléans & $/ 14$ \\
\hline 11 & Imprimerie Camis & 19 & 7 & Orléans \& Midi & 15 \\
\hline 12 & Imprimerie A. Bellier \& Cie & 19 & 4 & PLM & 110 \\
\hline 13 & Imprimerie Eug, Marx & 17 & 6 & Nord & 17 \\
\hline 14 & Éditions Mayeux & 17 & 4 & Étar & $/ 10$ \\
\hline 15 & Affiches (photographiques) Robaudy & 16 & 2 & PLM & 115 \\
\hline 16 & Imprimerie J. Minot & 14 & 5 & Orléans & 16 \\
\hline 17 & Imprimerie de Vaugirard & 14 & 5 & PLM & 111 \\
\hline 18 & Imprimerie Alsacienne & 13 & 2 & $\mathrm{AL}$ & $/ 12$ \\
\hline 19 & Éditions Paul Martial & 13 & 3 & \multicolumn{2}{|c|}{ Grands Réseaux français $/ 5$} \\
\hline 20 & Imprimerie E. Baudelot et Cie & 12 & 4 & Midi & 17 \\
\hline 21 & Imprimerie Lemercier et Cie & 11 & 8 & PLM & 14 \\
\hline 22 & Imprimerie Berger-Levrault \& Cie & 11 & 2 & PLM & $/ 10$ \\
\hline 23 & Imprimerie Baconnier Frères & 11 & 4 & PLM & 16 \\
\hline 24 & Imprimerie Rotophot & 10 & 2 & État & 19 \\
\hline 25 & Imprimerie Devambez & 10 & 4 & Midi-PLM & 14 \\
\hline 26 & Imprimerie Daude Frères & 10 & 3 & État & 15 \\
\hline
\end{tabular}

L'échantillon retenu est composé des 25 imprimeurs ayant réalisé plus de 10 affiches chacun pendant la période étudiée.

\section{Annexe 4}

Affiche de style graphique (20\% du corpus) - Dunkerque, 1929

\section{Nous ne sommes pas autorisés à reproduire cette image.}

Annexe 5 
Carte postale de style traditionnel (80\% du corpus) - L'Auvergne, 1893

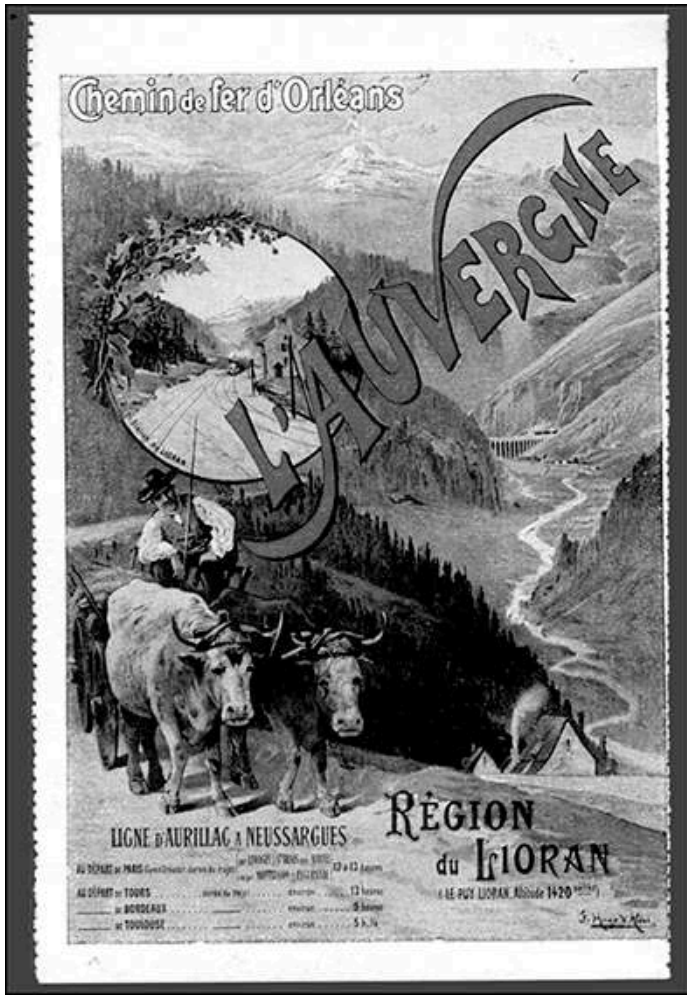

Illustrateur : Hugo d'Alési - Commanditaire : Chemin de fer d'Orléans - Imprimeur : A. Bellier et Cie Coll. et cl. : N. Pégé-Defendi

\section{Annexe 6}

Affiche et maquette (de g. à d.) - Berck-Plage, 1936
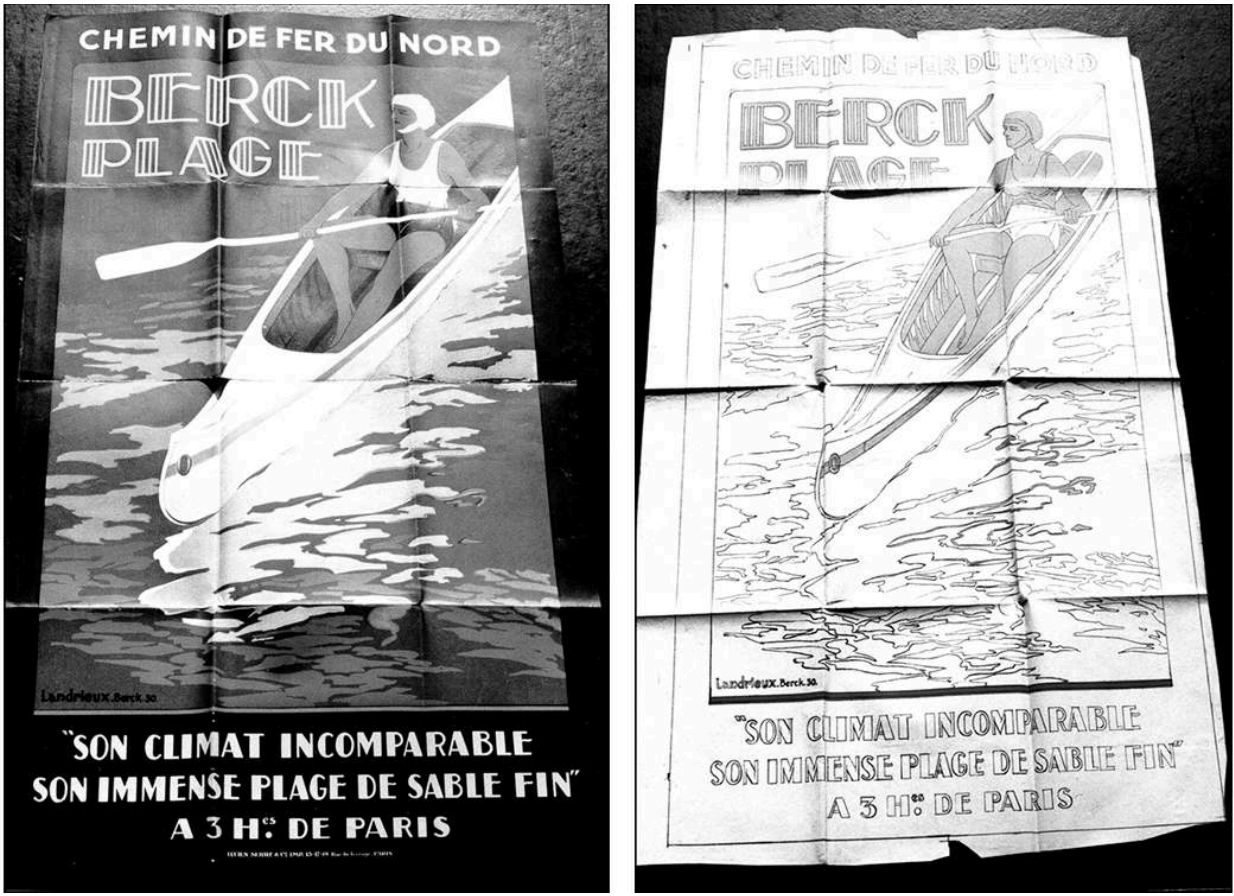

Coll. Archives Nationales (Centre des archives du monde du travail) - cl. N. Pégé-Defendi 


\section{NOTES}

1. Nathalie Pégé-Defendi, "Une invitation au tourisme: l'affiche ferroviaire française (1880-1936) », thèse pour le doctorat de l'université de Paris I - Panthéon-Sorbonne, juin 2001, 789 p. de texte en 2 vol., un vol. de planches (402 p.), soutenue par l'Association pour l'histoire des chemins de fer en France.

INDEX

Keywords : tourism, poster, railway, France

Mots-clés : tourisme, affiche, chemin de fer, France

\section{AUTEUR}

NATHALIE PÉGÉ-DEFENDI

Docteur en histoire 